\title{
Positronium chemistry studied by AMOC measurements using a relativistic positron beam
}

\author{
H. SCHNEIDER ${ }^{*}$ A. SEEGER ${ }^{*, * *}$, A. SIEGLE ${ }^{* *}$, H. STOLL ${ }^{*}$ I. BILLARD ${ }^{* *}$, M. KOCH*, \\ U. LAUFF* and J. MAJOR* \\ ${ }^{*}$ Max-Planck-Institut für Metallforschung, Institut für Physik, Heisenbergstr. 1, 70569 Stuttgart, Germany \\ ** Universität Stuttgart, Institut für Theoretische und Angewandte Physik, 70569 Stuttgart, Germany \\ *** Centre de Recherches Nucléaires, Laboratoire de Chimie Nucléaire, BP. 20, 67037 Strasbourg cedex, \\ France
}

\begin{abstract}
Beam-based Age-Momentum Correlation $\left(\beta^{+} \gamma \Delta E\right.$ AMOC) measurements using an $\mathrm{MeV}$ positron beam have become a powerful tool to study chemical reactions of positronium by time-domain observations of the different positron states tagged by the Doppler-broadening $(\Delta E)$ characteristics of the $511 \mathrm{keV}$ annihilation radiation. As an example, the investigation of the spin-conversion reaction in the system HTEMPO/methanol is reported. The experimental data for small HTEMPO concentrations can be fitted quite well to a rate-equation model containing the conversion rate as the only adjustable parameter. This model can presumably be applied not only to spin conversion but also to other chemical reactions and to inhibition of positronium. Possible effects of oxidation, complex formation, and inhibition are discussed by way of examples.
\end{abstract}

\section{Introduction and General Background}

Positron $\left(\mathrm{e}^{+}\right)$annihilation has become a powerful tool for studying physical properties or chemical reactions in condensed matter. One of the more recent developments is the possibility to investigate transitions between various positron states as a function of time. Examples are positron trapping at defects in solids (see, e.g., [1]) or chemical reactions of positronium $\mathrm{P}_{\mathrm{S}}=\left(\mathrm{e}^{+} \mathrm{e}^{-}\right)$("positronium chemistry" [2]).

Since $e^{+}-e^{+}$interactions are always negligible, the time dependence of the populations $n_{i}$ of the different positron states $i$ can be described by a system of linear differential equations that are of first order in time $t$. Usually it is assumed that they can be approximated by a system of rate equations:

$$
\frac{\mathrm{d} n_{i}(t)}{\mathrm{d} t}+\left(\tau_{i}^{-1}+\sum_{j=0, j \neq i}^{N} \kappa_{i j}\right) n_{i}(t)=\sum_{j=0, j \neq i}^{N} \kappa_{j i} n_{j}(t) \quad(i=0,1,2, \ldots, N) .
$$

Here $\tau_{i}$ denotes the $\mathrm{e}^{+}$lifetimes as defined by the annihilation in the state $i$ and $\kappa_{i j}$ the transition rate from state $i$ to state $j$. Starting the summation in (1) with $j=0$ allows us to attribute the subscript zero to a particularly simple reference state appearing in almost all applications, e.g., in the case of crystalline materials corresponding to $\mathrm{e}^{+}$Bloch waves in perfect crystals [3]. The lifetimes $\tau_{i}$ are determined by the overlap of the electron $\left(\mathrm{e}^{-}\right)$and $\mathrm{e}^{+}$wavefunctions and, in the case of Ps formation or of annihilation in magnetically ordered matter, by the spin distributions of $\mathrm{e}^{+}$and $\mathrm{e}^{-}$. The values of the $\tau_{i}$ of the various positron states as obtained from experiments can give us strong hints of the nature of these states. E.g., in vacuum the lifetimes of ortho- and para-Ps are $\tau_{\mathrm{o}-\mathrm{Ps}}^{\mathrm{v}}=140 \mathrm{~ns}$ and 
$\tau_{\mathrm{p}-\mathrm{Ps}}^{v}=125 \mathrm{ps}$. Therefore it is a clear indication of Ps formation if in a metallic system one of the lifetimes $\tau_{i}$ exceeds the value $\tau_{\infty}=4\left(1 / \tau_{\mathrm{p}-\mathrm{Ps}}^{\mathrm{v}}+3 / \tau_{\mathrm{o}-\mathrm{Ps}}^{\mathrm{v}}\right)^{-1} \approx 500 \mathrm{ps}$ [4]. A similar though somewhat larger limit holds for dielectric materials.

Diffusion-controlled reactions require special considerations. Positron trapping by isolated traps may still be described by (1) with $t$-dependent $\kappa_{i j}[5]$. In three-dimensional systems, these quantities approach at large $t$ finite asymptotic values $\kappa_{i j}(\infty)$. In this case the time dependence is confined to times (after positron thermalization) not much longer than $4 r_{i j}^{2} / \pi D$, where $D$ is the diffusivity controlling the transition (e.g., in the case of Ps trapping the sum of the trap diffusivity and the Ps diffusivity) and $r_{i j}$ the reaction radius, i.e., the distance to the reaction center the $\mathrm{e}^{+}(\mathrm{Ps})$ must approach in order to react [6]. An example of a diffusion-controlled $\mathrm{e}^{+}$reaction in which the timedependence of the rate coefficients does make itself clearly felt, viz. the trapping of hydrated $\mathrm{e}^{+}$by halide ions in aqueous solutions of alkali halides, has been studied by Mogensen et al. [7].

When all $\kappa_{i j}$ may be taken as time-independent, the solution of (1) may be written as

$$
n_{i}(t)=\sum_{j=0}^{N} A_{i j} \exp \left(-\Gamma_{j} t\right)
$$

where the "decay rates" $\Gamma_{j}$ are obtained as functions of the $\mathrm{e}^{+}$lifetimes $\tau_{i}$ and of the transition rates $\kappa_{i j}$ by solving a secular problem [3]. The weight factors $A_{i j}$ depend, in addition, on the "initial distribution" $n_{i}(0)$ describing the occupancy of the $\mathrm{e}^{+}$states at the end of the thermalization period that follows the $\mathrm{e}^{+}$implantation.

In other cases, e. $g$. the trapping of $\mathrm{e}^{+}$in grain boundaries $[8,9]$ or Ps reactions such as the one to be discussed in detail in Sect. 4, the theoretical treatment must be based on diffusion-reaction equations rather than rate equations such as (1). For simple models it is easy to obtain the Laplace transform of the age-dependence of the positron annihilation rate and also some average quantities such as the mean positron lifetime. However, the inversion of the Laplace transform can be done in closed form only in certain limiting cases. In general, (2) has to be replaced by an infinite series. If only a few terms (say, two or three) in these series are relevant, the results may be approximately described in terms of rate equations.

The two quantities which characterize the annihilation of a positron in condensed matter are the momentum $\boldsymbol{p}$ of the annihilating electron-positron pair and the positron age $t$, defined as the time interval between the implantation and the annihilation of the positron. Since the two $511 \mathrm{keV}$ photons resulting from a $2 \gamma$-annihilation event transmit equivalent information, one photon may be used to determine the age of the annihilating positron and the other one to deduce information on the momentum of the annihilating $\mathrm{e}^{+} \mathrm{e}^{-}$pair. This information may be obtained either in an ACAR (= Angular Correlation of Annihilation Radiation) arrangement or by measuring the distribution of Doppler shifts $(\Delta E)$ in a, say, Ge detector. The Doppler broadening of the $511 \mathrm{keV}$ annihilation radiation allows us to deduce the distribution function of the $\mathrm{e}^{+} \mathrm{e}^{-}$momenta parallel to the axis of the $\gamma$ emission ("longitudinal momentum") whereas ACAR measurements give us (with much better resolution than $\Delta E$ measurements) one or both momentum components perpendicular to the axis of $\gamma$ emission ("transverse momentum"). In the latter case, however, the same $\gamma$ quantum has to be used for both the stop signal of the age determination and for measuring the non-collinearity of the $2 \gamma$ emission in the laboratory system. Since the requirements of these two measurements are to not a small degree in conflict with each other, for most purposes it is best to deduce the momentum information required for AMOC from the Doppler broadening of the $511 \mathrm{keV}$ annihilation line.

The simultaneous detection of the two $511 \mathrm{keV}$ annihilation $\gamma$ quanta in an $\mathrm{e}^{+}$lifetime spectrometer and in a $\Delta E$ (= Doppler-broadening) or ACAR set-up permits us to establish the correlation between the $\mathrm{e}^{+}$ages (= individual $\mathrm{e}^{+}$lifetimes) and the momentum components of the annihilating $\mathrm{e}^{+} \mathrm{e}^{-}$pairs. This AMOC (= Age-Momentum-Correlation) technique is an extremely powerful tool for the study of reactions involving positrons. It not only provides the information obtainable from the two constituent measurements ( $\mathrm{e}^{+}$lifetime spectroscopy and determination of the $\mathrm{e}^{+} \mathrm{e}^{--}$ momentum distribution) but also allows us to follow directly, in the time domain, the change in the 
$\mathrm{e}^{+} \mathrm{e}^{-}$-momentum distribution associated with the transitions between different positron states.

Hsu and Wu [10] and McGervey and Walters [11] demonstrated the existence of a correlation between $\mathrm{e}^{+}$age and $\mathrm{e}^{+} \mathrm{e}^{-}$momentum by performing lifetime measurements with annihilation quanta of selected non-collinearities. Furthermore, Hsu and $\mathrm{Wu}[10]$ obtained separate Doppler-broadened $511 \mathrm{keV}$ lines for young and old $\mathrm{e}^{+}$, i.e., they initiated the technique now called TSES (= TimeSelected Energy Spectrum) [12]. The pioneering work on AMOC in the narrower sense was carried out by MacKenzie and collaborators [13-15], employing Doppler broadening, followed by the investigations of Tanigawa and collaborators [16-21]. For a recent attempt to make use of the high momentum resolution of ACAR in AMOC studies see Saito et al. [22].

The present paper reviews recent AMOC studies $[23,24]$ on the room-temperature Ps spinconversion reaction in the system methanol/HTEMPO (4-hydroxy-2,2,6,6-tetramethylpiperidine1-oxyl), a reaction that was investigated previously by separate lifetime and Doppler-broadening measurements [25]. Sect. 2 gives an outline of the special $\triangle E$ AMOC technique used in the present and other (see, e.g., [26-28]) recent work. Sect. 3 deals with Ps in pure methanol. Sect. 4 discusses the drastic modifications of the room-temperature age-momentum correlation that are induced by relatively small additions of HTEMPO. As will be seen, they can almost entirely be accounted for by Ps spin conversion but other processes may also play a rôle.

\section{Age-Momentum-Correlation (AMOC) Measurements}

The great potential of AMOC has to be seen against the background of a number of practical obstacles. Since AMOC measurements necessarily involve triple coincidences between the (delayed) start signal for the age measurement and the signals generated by the two annihilation $\gamma$ quanta, the low detector efficiency of $\gamma$ detectors makes the data accumulation rather slow. Obtaining good statistics within a reasonable time presents a problem. As long as the conventional technique of using the emission of prompt $\gamma$ quanta from the $\mathrm{e}^{+}$source (e.g., the $1275 \mathrm{keV}$ photons from ${ }^{22} \mathrm{Na}$ ) for generating the start signal (called $\gamma \gamma$ coincidence technique) is employed, the problem cannot be solved by means of stronger and stronger sources. The triple-coincidence rate achievable in such a set-up is limited by the deterioration of the spectra due to random coincidences of signals generated by positrons that are implanted in the sample almost simultaneously.

In very early positron lifetime measurements [29] the start signal was generated not by a prompt $\gamma$ quantum but by the detection of the positron emission. Since the detection efficiency of $\beta^{+}$detectors is almost unity, a $\beta^{+} \gamma$ coincidence technique is basically superior to the $\gamma \gamma$ coincidence technique with regard to the coincidence counting rate. Source-based $\beta^{+} \gamma \Delta E$ AMOC measurements can indeed be performed very easily in samples acting as scintillators [14]. However, if the $\beta^{+}$scintillator has to be separated from the sample, the time-of-flight jitter of positrons of different energies has to be considered, unless the distance between the scintillator and the sample can be made very small $[30,31]$. The elimination of the time jitter by placing a $\beta^{+}$spectrometer between the $\beta^{+}$detector and the sample [29] reduces the rate of $\mathrm{e}^{+}$implantation seriously.

In 1978 Maier and Myllylä [32] suggested that the problem of time jitter in the $\beta^{+} \gamma$ coincidence technique can be eliminated by employing relativistic $\mathrm{e}^{+}$whose speed is close to the velocity of light and therefore virtually unaffected by energy losses in the start detector. The AMOC measurements discussed in this review were made possible by a unique combination of features of the beam-based $\beta^{+} \gamma \Delta E$ AMOC facility at the Max-Planck-Institut für Metallforschung, Stuttgart [33-35]. The most important of these are:

i) The start signal for the age determination is created not by a prompt $\gamma$ quantum but in a plastic-scintillator $\beta^{+}$counter through which the $\mathrm{e}^{+}$have to pass before implantation. This gives a detection efficiency very close to unity.

ii) Pile-up pulses in the $\beta^{+}$counter are used to discriminate efficiently against multiple $\mathrm{e}^{+}$annihilation. This allows $\mathrm{e}^{+}$to be implanted into the sample at very high rates without the above-mentioned limitation of the conventional set-up.

iii) The information on the $\mathrm{e}^{+} \mathrm{e}^{-}$-momentum distribution is derived using a Doppler-broadening measurement by means of a Ge detector (FWHM $=1.4 \mathrm{keV}$ ). 

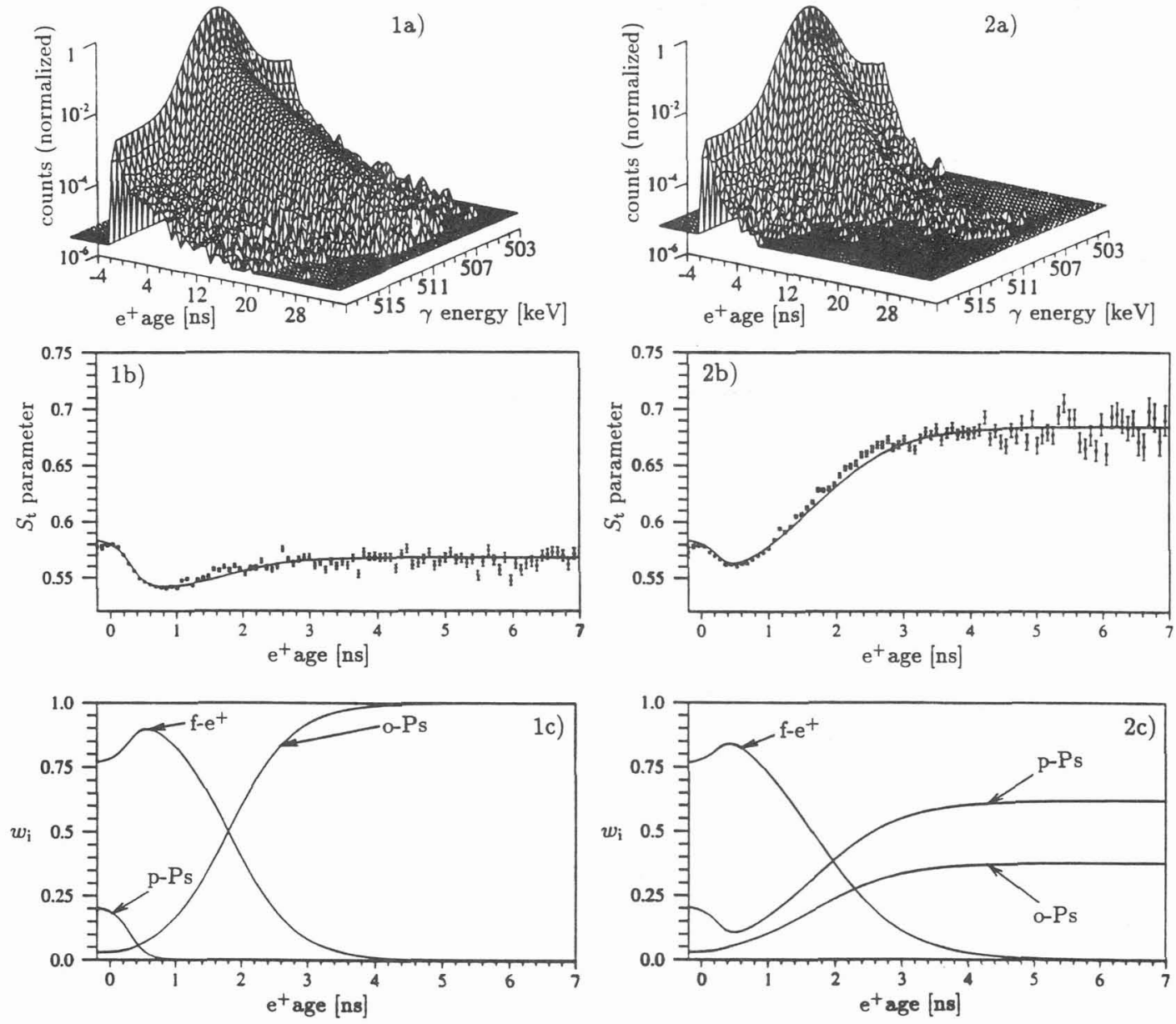

Fig. 1: a) $\beta^{+} \gamma \Delta E$ AMOC relief of methanol at room temperature. b) Lineshape parameter $S_{\mathrm{t}}$ vs. positron age derived from the AMOC spectrum. The solid curve represents a fit to the rate-equation model of Sect.3. c) Normalized annihilation events $w_{i}$ vs. $\mathrm{e}^{+}$age as determined from the fit.

Fig. 2: a) $\beta^{+} \gamma \Delta E$ AMOC relief of $0.1 \mathrm{~mol} / 1$ HTEMPO in methanol (room temperature). b) $S_{t}$ vs. positron age for $0.1 \mathrm{~mol} / 1$ HTEMPO in methanol and fit to the rate-equation model of Sect.4. c) Normalized annihilation events $w_{i}$ vs. $\mathrm{e}^{+}$age as determined from the fit.

Examples of "AMOC reliefs" (logarithm of the number of counts as a function of $\mathrm{e}^{+}$age and $\gamma$ energy) are shown in Figs. 1a and 2a. Cuts along planes of constant energy represent lifetime spectra with different longitudinal momenta of the annihilating $\mathrm{e}^{+} \mathrm{e}^{-}$pair, cuts along planes of constant time present energy spectra at different $\mathrm{e}^{+}$ages. The longitudinal momentum distribution associated with the $\mathrm{e}^{+}$state $i$ may be characterized by the lineshape parameter $S_{i}$ [36] defined by the number of counts in a central region (in the measurements of Sect. $31.85 \mathrm{keV}$ wide) of the Doppler-broadened $511 \mathrm{keV}$ line normalized by the total number of counts in this line. The age-dependent total lineshape parameter $S_{\mathrm{t}}(t)$, a quantity that may be experimentally determined with good statistical significance, is a linear combination of the partial lineshape parameter $S_{i}$ weighted by the normalized annihilation events $w_{i}$ :

$$
S_{\mathrm{t}}(t)=\sum_{i=0}^{N} S_{i} w_{i}(t):=\sum_{i=0}^{N} S_{i} \frac{n_{i}(t) \tau_{i}^{-1}}{\sum_{j=0}^{N} n_{j}(t) \tau_{j}^{-1}} .
$$

Figs. $1 \mathrm{~b}$ and $2 \mathrm{~b}$ give examples of the age dependence of $S_{\mathrm{t}}(t)$. Note that large $\mathrm{S}$ parameters denote 
narrow lines and therefore narrow momentum distributions of the $\mathrm{e}^{+} \mathrm{e}^{-}$pairs.

\section{AMOC Study of Ps Formation in Methanol}

Fig. 1a shows the two-dimensional age-momentum correlation relief of methanol at room temperature. $S_{\mathrm{t}}(t)$ (Fig. 1b) may be fitted by (2) and (3) with $N=2(i=0,1,2)$. In agreement with previous resuits $[37,38]$ the three positron states are attributed to free positrons ( $\left(\mathrm{f}^{+}\right)$, para-positronium (p-Ps), and ortho-positronium (o-Ps). The parameters $S_{i}, \tau_{i}$, and the normalized initial population obtained from a quantitative fit to the data (solid line in Fig. $1 \mathrm{~b}$ ) are given in Table 1.

Table 1: Parameters for the different positron states ( $\left(-e^{+}, p-P s, o-P s\right.$, ) resulting from a fit of Eqns. (2) and (3) to the measured $S_{t}(t)$ of methanol at room temperature (Fig. 1b).

\begin{tabular}{|r|lll|}
\hline positron state $i$ & f-e $^{+}$ & p-Ps & o-Ps \\
\hline partial lineshape parameter $S_{i}$ & 0.54 & 0.75 & 0.57 \\
positron lifetime $\tau_{i}[\mathrm{ps}]$ & 450 & 125 & 3500 \\
normalized initial population & 0.732 & 0.067 & 0.201 \\
\hline
\end{tabular}

Figure 1c shows the normalized annihilation events of the different positron states $i$ as a function of positron age. The assignments of Fig. 1c are based on the following arguments. At $\mathrm{e}^{+}$ages exceeding 3 ns almost all positrons annihilate in the longest-lived $\mathrm{e}^{+}$state, viz. o-Ps. The positrons of o-Ps mainly undergo $2 \gamma$ "pick-off" annihilation with "foreign" electrons. The "old-age" $S_{t}$ parameter (Fig. 1b) is dominated by the momentum distribution of these electrons. The fact that in the age interval $0.3-2 \mathrm{~ns} S_{\mathrm{t}}$ is smaller than this value (exhibiting a minimum at about $0.7 \mathrm{~ns}$ ) is attributed to the annihilation of $\mathrm{f}-\mathrm{e}^{+}$, which shows a slightly broader momentum distribution than pick-off annihilation of the $\mathrm{e}^{+}$in o-Ps $\left(S_{\mathrm{f}-\mathrm{e}^{+}}<S_{\mathrm{o}-\mathrm{Ps}}\right.$, cf. Table 1$)$. At ages shorter than about $0.7 \mathrm{~ns}$ the influence of the $\mathrm{f}-\mathrm{e}^{+}$annihilation is counteracted by the annihilation of $\mathrm{p}-\mathrm{Ps}$, which is not only very short-lived but also has by far the largest $S_{i}$ parameter of the three states considered. As is well known, this is due to the fact that $\mathrm{e}^{+}$in p-Ps annihilates with its "own" electron; in this case the $\mathrm{e}^{+} \mathrm{e}^{-}$momentum is determined by Maxwell's velocity distribution and hence very narrow compared with the Fermi momentum distribution of the valence electrons contributing to $S_{\mathrm{f}-\mathrm{e}^{+}}$and $S_{\mathrm{o}-\mathrm{Ps}}$.

\section{Spin-Conversion of Ps in the System Methanol/HTEMPO}

The addition of $0.1 \mathrm{~mol} / 1 \mathrm{HTEMPO}$ to methanol changes drastically the AMOC relief and the age dependence of the lineshape parameter $S_{\mathrm{t}}$, as comparison between Figs. 2a-c and Figs. 1a-c shows. This is attributed to the spin-conversion reactions

$$
\begin{aligned}
& \mathrm{o}-\mathrm{Ps}+\mathrm{M} \longrightarrow \frac{1}{4} \mathrm{p}-\mathrm{Ps}+\frac{3}{4} \mathrm{o}-\mathrm{Ps}+\mathrm{M} \\
& \mathrm{p}-\mathrm{Ps}+\mathrm{M} \longrightarrow \frac{1}{4} \mathrm{p}-\mathrm{Ps}+\frac{3}{4} \mathrm{o}-\mathrm{Ps}+\mathrm{M}
\end{aligned}
$$

between the Ps "atoms" and the paramagnetic HTEMPO molecules (M), for which the unpaired electron in the nitrosyl group of HTEMPO is thought to be responsible [25]. Reaction (4) counteracts the depletion of the p-Ps population caused by the short p-Ps lifetime and tends to retain the initial 1:3 ratio between the p-Ps and o-Ps populations. The opening of an additional decay channel of o-Ps by the spin-conversion to p-Ps results in a shortening of the o-Ps lifetime (cf. Fig. 1a and Fig. 2a) and causes short-lived p-Ps with its narrow annihilation line to make a significant contribution to the AMOC spectrum also at high $\mathrm{e}^{+}$positron ages (Fig. $2 \mathrm{~b}$ ). The lineshape parameters $S_{\mathrm{t}}(t)$ at ages exceeding 3 ns are determined by the ratio of the annihilation events of o-Ps and of spin-converted p-Ps (cf. Fig. 2c) and thus by the HTEMPO concentration $C$.

As mentioned in Sect. 1, a proper treatment of the effects of reactions such as (4) on positron annihilation has to be based on diffusion-reaction equations. For a simple but presumably adequate model a full solution has recently been given [39]. The model describes the $\mathrm{M}$ species by little spheres, inside of which Ps may undergo the spin-conversion reactions (4). The Ps "atoms" have to reach these little spheres by diffusion. The complete solution of the problem is too complicated to be reported here. As one expects from intuitive reasoning, the $\mathrm{e}^{+}$decay rates depend on the positron 
diffusivity $D^{+}$only if $D^{+}$is neither very small nor very large. In the intermediate range it should be possible to determine $D^{+}$from measurements. In the limit of large diffusivities and small $\mathrm{M}$ concentrations but arbitrary reaction-rate constant $k_{\text {conv }}$ (= specific reaction rate) the results may be represented by Eqn. (2) with $N=2$, viz. a free-electron component and two Ps components, with

$$
\Gamma_{0}=\tau_{\mathrm{f}-\mathrm{e}^{+}}^{-1}, \Gamma_{1,2}=\frac{1}{2}\left\{\tau_{\mathrm{p}-\mathrm{Ps}}^{-1}+\tau_{\mathrm{o}-\mathrm{Ps}}^{-1}+k_{\mathrm{conv}} C \pm\left[\left(\tau_{\mathrm{p}-\mathrm{Ps}}^{-1}-\tau_{\mathrm{o}-\mathrm{Ps}}^{-1}+\frac{1}{2} k_{\mathrm{conv}} C\right)^{2}+\frac{3}{4} k_{\mathrm{conv}}^{2} C^{2}\right]^{\frac{1}{2}}\right\}
$$

Fits of a rate-equation model that is fully compatible with Eqns. (5) to room-temperature $\beta^{+} \gamma \Delta E$ AMOC measurements on different HTEMPO concentrations in methanol up to $0.1 \mathrm{~mol} / 1$ gave a linear relationship

$$
K_{\text {conv }}=k_{\text {conv }} C
$$

between the spin-conversion reaction rate $K_{\text {conv }}$ and the HTEMPO concentration $C$ [23]. In these fits the only concentration-dependent parameter was the reaction rate $K_{\text {conv. }}$. All other parameters were kept at values determined from the fit of the pure solvent (see Table 1). The reaction-rate constant $k_{\text {conv }}=(22.5 \pm 0.5) \cdot 10^{9} \mathrm{l} \mathrm{mol}^{-1} \mathrm{~s}^{-1}$ obtained in this way [23] is in satisfactory agreement with $k_{\text {conv }}=19.2 \cdot 10^{9} \mathrm{Imol}^{-1} \mathrm{~s}^{-1}$ as deduced from separate lifetime-spectroscopy and Dopplerbroadening measurements $[25,38]$. Fig. $2 \mathrm{~b}$ shows that in the case of $C=0.1 \mathrm{~mol} / 1$ the fit to the rate-equation model is not perfect, however. Reasons for this may be unjustified simplifications by the use of the rate-equation model, a slight concentration dependence of the parameters listed in Table 1 , or the influence of additional reactions.

A qualitative understanding of the effects of other reactions may be obtained from Fig. 3, where examples of $S_{\mathrm{t}}$ parameters as a function of positron age calculated by means of a rate-equation model [24] are shown for a) spin-conversion, b) oxidation, c) complex formation, and d) inhibition of positronium (dashed lines). The solid lines represent the $S_{\mathfrak{t}}(t)$ parameter of the solvent (methanol).
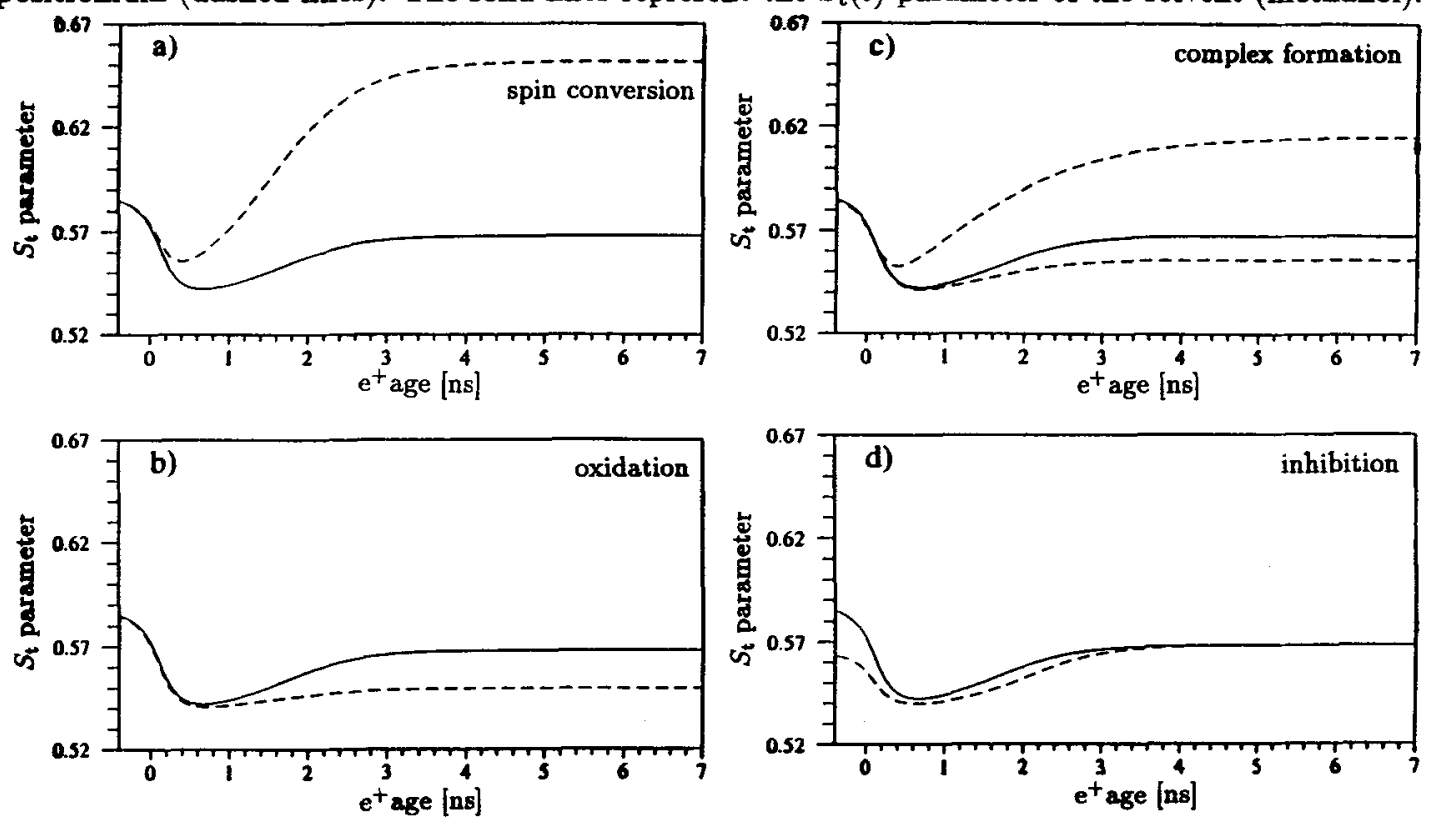

Fig. 3: Calculated $S_{\mathrm{t}}(t)$ (dashed lines) for a) spin-conversion $\left(K_{\text {conv }}=10^{9} \mathrm{~s}^{-1}\right)$, b) oxidation $\left(K_{\mathrm{ox}}=\right.$ $\left.0.3 \cdot 10^{9} \mathrm{~s}^{-1}\right)$, c) complex formation $\left(S_{\text {compl }}=0.62\right.$ (upper line) or $S_{\text {compl }}=0.53$ (lower line) with $\tau_{\text {compl }}=$ $530 \mathrm{ps}, K_{\text {compl }}=10^{9} \mathrm{~s}^{-1}$ ), and d) inhibition of positronium (initial positronium fraction $13 \%$ compared to $27 \%$ in methanol). The solid lines in the figures represent $S_{t}(t)$ of the solvent (methanol).

Oxidation generates free positrons from positronium states. In common with the spin-conversion reaction discussed above (Fig. $2 \mathrm{~b}$ and $3 \mathrm{a}$ ), oxidation affects the $S_{\mathrm{t}}(t)$ particularly at high positron ages 
(Fig. 3b). The lineshape parameter $S_{\mathrm{t}}(t>3 \mathrm{~ns})$ is now determined by the ratio of the contributions of $\mathrm{o}-\mathrm{Ps}$ and free $\mathrm{e}^{+}$to the positron annihilation (cf. Eqn. (3)). Since in methanol the free $\mathrm{e}^{+}$annihilation leads to stronger Doppler broadening than the pick-off annihilation of o-Ps (cf. Table 1) the $S_{\mathrm{t}}(t)$ parameter at high ages is lowered by oxidation.

Complex formation generates a fourth positron state, viz. Ps bound to a molecule. Therefore three additional parameters have to be considered: the initial population $n_{\text {compi }}(0)$, the lineshape parameter $S_{\text {compl }}$, and the $\mathrm{e}^{+}$lifetime $\tau_{\text {compl. }}$. The examples of complex forming reactions shown in Fig. $3 c$ are based on the initial condition $n_{\text {compl }}(0)=0$.

Inhibition and anti-inhibition affect the probability of positronium formation and thus the initial population $n_{i}(0)$ of the positron states. If the formation of positronium is inhibited, the enhanced annihilation of free positrons decreases the $S_{t}(t)$ parameter, particularly at short positron ages (Fig. 3d). Recent AMOC measurements on HTEMPO concentrations higher than $0.1 \mathrm{~mol} / 1$ indicate that inhibition might have to be considered in the HTEMPO/methanol system.

\section{Conclusions}

Age-Momentum Correlation (AMOC) measurements are a powerful and versatile technique for studying reactions involving positrons and positronium in condensed matter. A $\beta^{+} \gamma \Delta E$ AMOC facility $\left(\beta^{+} \gamma\right.$ : positron lifetime spectroscopy using a $\beta^{+}$scintillator with high detection efficiency for generating the start signal and one of the $511 \mathrm{keV}$ annihilation $\gamma$-quanta to generate the stop signal; $\Delta E$ : measurement of the Doppler broadening of the $511 \mathrm{keV}$ annihilation line using the second annihilation $\gamma$-quantum) based on a relativistic $\mathrm{e}^{+}$beam $\left(E_{\mathrm{kin}}^{\mathrm{e}^{+}} \approx 4 \mathrm{MeV}\right)$ at the Stuttgart Pelletron allows AMOC measurements to be performed routinely. A parameter characterizing the momentum distribution of the $\mathrm{e}^{+} \mathrm{e}^{-}$pairs annihilating in the various positron states (e.g., the lineshape parameter $S_{\mathrm{t}}(t)$ ) can be deduced as a function of the positron age $t$ (= time interval between implantation and annihilation of the individual positrons) and permits us to follow the transition of positrons between different states rather directly.

\section{Acknowledgments}

Co-operation and helpful discussions with J. Ch. Abbé, G. Duplâtre, K. Maier, and R. Würschum are gratefully acknowledged. The authors are indebted to all members of the Stuttgart positron group as well as to the staff of the Stuttgart Pelletron for their ongoing support. They should also like to thank A. Bonnenfant (Strasbourg) and J. Sprater (MPI für Festkörperforschung, Stuttgart) for preparing the methanol and HTEMPO samples.

\section{References}

[1] A. Seeger and F. Banhart, Helv. Phys. Acta 63, 403 (1990).

[2] D. M. Schrader and Y. C. Jean (Eds.), "Positron and Positronium Chemistry", Elsevier, Amsterdam, 1988.

[3] A. Seeger, Appl. Phys. 4, 183 (1974).

[4] A. Seeger and F. Banhart, Phys. Stat. Sol. (a) 102, 171 (1987)

[5] W. Frank and A. Seeger, Appl. Phys. 3, 61 (1974).

[6] A. Seeger, in "Frontiers in Materials Science: Distinguished Lectures", Eds. L. Murr and Ch. Stein (Marcel Dekker Inc., New York 1974) p. 177

[7] O. E. Mogensen, M. Eldrup, and H. J. Pedersen, in "Positron Annihilation", Eds. P. C. Jain, R. M. Singru, and K. P. Gopinathan (World Scientific, Singapore 1985) p. 756.

[8] A. Seeger, Mater. Sci. Forum 105-110, 821 (1988)

[9] A. Seeger and R. Würschum, to be published

[10] F. H. H. Hsu and C. S. Wu, Phys. Rev. Lett. 18, 889 (1967).

[11] J. D. McGervey and V. F. Walters, Phys. Rev. B2, 2421 (1970). 
[12] T. B. Chang, G. M. Yang, and T. Hyodo, Mater. Sci. Forum 105-110, 1509 (1992).

[13] I. K. MacKenzie and P. Sen, Phys. Rev. Lett. 37, 1296 (1976).

[14] I. K. MacKenzie and B. T. A. McKee, Appl. Phys. 10, 245 (1976).

[15] S. Linderoth and I. K. MacKenzie, in "Positron Annihilation", Eds. P. C. Jain, R. M. Singru, and K. P. Gopinathan (World Scientific, Singapore 1985) p. 833.

[16] S. Tanigawa, Y. Kishimoto, and N. Tsuda, in "Positron Annihilation", Eds. P. G. Coleman, S. C. Sharma, and L. M. Diana (North Holland, Amsterdam 1982) p. 654.

[17] S. Tanigawa, K. Shima, H. Irijama, and Y. Waseda, in "Positron Annihilation", Eds. P. G. Coleman, S. C. Sharma, and L. M. Diana (North Holland, Amsterdam 1982) p. 584.

[18] Y. Kishimoto, S. Tanigawa, H. Morinaga, and Y. Matsuoka, in "Positron Annihilation", Eds. P. G. Coleman, S. C. Sharma, and L. M. Diana (North Holland, Amsterdam 1982) p. 168.

[19] Y. Kishimoto, K. Ito, S. Tanigawa, and N. Tsuda, in "Positron Annihilation", Eds. P. G. Coleman, S. C. Sharma, and L. M. Diana (North Holland, Amsterdam 1982) p. 843.

[20] Y. Kishimoto and S. Tanigawa, in: "Positron Annihilation", Eds. P. G. Coleman, S. C. Sharma, and L. M. Diana (North Holland, Amsterdam 1982) pp. 404, 790, 815.

[21] Y. Iwase, A. Uedono, S. Tanigawa, and H. Sakairi, in "Positron Annihilation", Eds. P. C. Jain, R. M. Singru, and K. P. Gopinathan (World Scientific, Singapore 1985) p. 868.

[22] H. Saito, Y. Nagashima, and T. Hyodo, Mater. Sci. Forum, 105-110 1967, (1992)

[23] H. Stoll, M. Koch, U Lauff, K. Maier, J. Major, A. Seeger, P. Wesołowski, I. Billard, J. Ch. Ábbé, G. Duplâtre, S. H. Connell, J. P. F. Sellschop, E. Sideras-Haddad, K. Bharuth-Ram, and H. Haricharun, Proceedings of the $5^{\text {th }}$ Intern. Workshop on Slow-Positron Beam Techniques for Solids and Surfaces, Jackson, Wyoming, USA, 1992, to be published in the AIP Proceedings Series.

[24] U. Lauff, K. Maier, J. Major, A. Seeger, H. Stoll, P. Wesołowski, M. Koch, I. Billard, J. Ch. Abbé, and G. Duplâtre, Acta Phys. Pol. A 83, 349 (1993).

[25] J. Ch. Abbé, G. Duplâtre, A. Haessler, A. Marques Netto, and D. Pilo Veloso, J. Phys. Chem. 88, 2071 (1984).

[26] H. Stoll, M. Koch, K. Maier, and J. Major, Nucl. Instr. and Meth. B56/57, 582 (1991).

[27] H. Stoll, P. Wesołowski, M. Koch, K. Maier, J. Major, and A. Seeger, Mater. Sci. Forum 105-110, 1989 (1992).

[28] M. Koch, K. Majer, J. Major, A. Seeger, J. P. F. Sellschop, E. Sideras-Haddad, H. Stoll, and S. H. Connell, Mater. Sci. Forum 105-110, 671 (1992).

[29] R. E. Bell and R. L. Graham, Phys. Rev. 90, 644 (1953).

[30] J. Kostamovaara, M. Karras, and R. Myllylä, in "Positron Annihilation", Eds. P. G. Coleman, S. C. Sharma, and L. M. Diana (North Holland, Amsterdam 1982) p. 868.

[31] J. Kostamovaara, M. Karras, and R. Myllylä, Nucl. Instr. and Meth. B5, 80 (1984).

[32] K. Maier and R. Myllylä, in "Positron Annihilation", Eds. R. R. Hasiguti and K. Fujiwara, (Japan Institute of Materials, Sendai 1978) p. 829.

[33] W. Bauer, K. Maier, J. Major, H.-E. Schaefer, A. Seeger, H.-D. Carstanjen, W. Decker, J. Diehl, and H. Stoll, Appl. Phys. A43, 261 (1987).

[34] W. Bauer, J. Briggmann, H.-D. Carstanjen, S. Connell, W. Decker, J. Diehl, K. Maier, J. Major, H.-E. Schaefer, A. Seeger, H. Stoll, and E. Widmann, Nucl. Instr. and Meth. B50, 300 (1990).

[35] H. Stoll, M. Koch, K. Maier, and J. Major, Nucl. Instr. and Meth. B56/57, 582 (1991).

[36] I. K. MacKenzie, J. A. Eady, and R. R. Gingerich, Phys. Lett. 33A, 279 (1970).

[37] O. E. Mogensen and F. M. Jacobsen, Chem. Phys. 73, 223 (1982)

[38] I. Billard, J. Ch. Abbé, and G. Duplâtre, J. Phys. Chem. 95, 2501 (1991)

[39] R. Würschum and A. Seeger, to be published 